\title{
Page de titre
}

\section{Titre en français :}

Prise en charge palliative des patients allogreffés : recommandations de la Société Francophone de Greffe de Moelle et de Thérapie Cellulaire (SFGM-TC)

\section{Titre en anglais :}

Palliative care in hematopoietic stem-cell transplanted patients: guidelines from the Francophone Society of Bone Marrow Transplantation and Cellular Therapy (SFGM-TC)

Auteurs : Polomeni A, Prod'homme C, Ainaoui M, Bellec A, Berr A, Bonneau J, Charbonnier A, Coiteux V, de Berranger E, Descamps T, Gire M, Goncalves M, Ruscassié A, Yakoub-Agha I, Borel C.

\section{Affiliations :}

1) Alice Polomeni, Service d'hématologie clinique et thérapie cellulaire, Hôpital Saint Antoine, AP-HP, 184 rue du Faubourg Saint-Antoine 75011 Paris, alice.polomeni@aphp.fr

2) Chloé Prod'homme, Clinique de médecine palliative, Hôpital Calmette, CHRU de Lille, 2 avenue Oscar Lambret, 59037 Lille, chloe.prodhomme@chru-lille.fr

3) Malika Ainaoui, Service des maladies du sang, CHRU de Lille, avenue Michel Polonovski, 59037 Lille Cedex, malika.ainaoui@chru-lille.fr

4) Adeline Bellec, Service d'hématologie clinique, CHU de Rennes, 2 rue Henri Le Guilloux, 35033 Cedex 9, Rennes, adelinebellec@yahoo.fr

5) Aurélie Berr, Service d'hématologie, Institut de Cancérologie Strasbourg Europe, 17 rue Albert Calmette, BP 23025, 67033 Strasbourg Cedex a.berr@icans.eu

6) Jacinthe Bonneau, Service d'hématologie oncologie et greffe de moelle pédiatrique, CHU de Rennes, Hôpital Sud, 16 boulevard de Bulgarie, 35203 Rennes Cedex 2 ذacinthe.bonneau@chu-rennes.fr

7) Amandine Charbonnier, service d'hématologie clinique et thérapie cellulaire, CHUgroupe hospitalier Amiens-Sud, 90054 Amiens Cedex 1, charbonnier.amandine@chuamiens.fr

8) Valérie Coiteux, service des maladies du sang, CHRU de Lille, avenue Michel

Polonovski, 59037 Lille Cedex, valerie.coiteux@chru-lille.fr

9) Eva de Berranger, hématologie pédiatrique, hôpital Jeanne de Flandres, CHRU de Lille, Avenue Eugène Avinée, 59037 Lille Cedex, eva.deberranger@chru-lille.fr

10) Thomas Descamps, service des maladies du sang, CHRU de Lille, avenue Michel Polonovski, 59037 Lille Cedex, thomas.descamps@chru-lille.fr

11) Marion Gire, service d'hématologie, institut de cancérologie Lucien Neuwirth, 104 avenue Albert Raymond, 42270 Saint-Priest-en-Jarez, mariongire@gmail.com

12) Murielle Goncalves, service d'hématologie clinique et thérapie cellulaire, hôpital Saint Antoine, A.P.H.P., 184 rue du Faubourg Saint-Antoine 75011 Paris, murielle.goncalves@aphp.fr

13) Agnès Ruscassié, département des soins de support, institut universitaire du cancer de Toulouse, 1 avenue Irène Joliot-Curie, 31059 Toulouse Cedex ruscassie.agnes@iuctoncopole.fr

14) Ibrahim Yakoub-Agha CHU de Lille, LIRIC, INSERM U995 Université de Lille, 59000, Lille, France, Ibrahim.yakoubagha@chru-lille.fr 
15) Cécile Borel, service d'hématologie, institut universitaire du cancer de Toulouse, 1 avenue Irène Joliot-Curie, 31059 Toulouse Cedex borel.cecile@iuct-oncopole.fr

\title{
Auteur correspondant :
}

Nom et prénom : POLOMENI Alice

Adresse : Service d'hématologie clinique et thérapie cellulaire, Hôpital Saint Antoine, APHP, 184 rue du Faubourg Saint-Antoine 75011 Paris, alice.polomeni@aphp.fr

Téléphone : 0149283438

Prise en charge palliative de patients allogreffés : recommandations de la Société Francophone de Greffe de moelle et thérapie cellulaire (SFGM-TC)

Palliative care in hematopoietic stem-cell transplanted patients: guidelines from the Francophone Society of Bone Marrow Transplantation and Cellular Therapy (SFGM-TC)

\section{Résumé}

L'allogreffe de cellules souches hématopoïétiques, seule perspective curative pour certaines hémopathies malignes, comporte des risques de morbi-mortalité importants. Les attentes des patients et de leurs proches vis-à-vis de la procédure, les incertitudes pronostiques, ainsi que l'existence de nouvelles possibilités thérapeutiques, engendrent un recours fréquent, onéreux et pas toujours pertinent, aux soins intensifs. Favorisant une réflexion sur la proportionnalité des soins et sur la qualité de vie des patients, les soins palliatifs peuvent contribuer à la prise en charge des patients allogreffés et à l'accompagnement de leurs proches. Néanmoins, des obstacles à cette approche demeurent. Cet article a pour objectif de proposer des recommandations pour favoriser l'intégration de la démarche palliative au sein des unités d'allogreffe.

Mots clés : allogreffe ; allogreffe pédiatrique ; soins palliatifs ; pluridisciplinarité.

\begin{abstract}
Hematopoietic stem-cell transplantation (HCT), the only curative perspective for certain hematological malignancies, carries a significant risk of morbidity-mortality. The patients and families' expectations regarding the procedure, the prognosis uncertainties, as well as the existence of new therapeutic possibilities, lead to frequent, expensive and
\end{abstract}


not always relevant recourse to intensive care. By promoting reflection on the proportionality of care and the patients' quality of life, palliative care may contribute to the HCT patients management. Nevertheless, obstacles to this approach remain. The objective of this article is to propose recommendations to promote the integration of the palliative care within HCT units.

Key words: hematopoietic celle transplantation; pallaitive care, multidisciplinary approach.

\section{Etat de la question}

\section{$\underline{\text { Soins palliatifs en Hématologie }}$}

La plupart des patients souffrant d'hémopathies malignes reçoivent, au cours des derniers mois de vie, des chimiothérapies et/ou thérapies ciblées, ont souvent recours aux urgences, aux hospitalisations et à la réanimation. Ils bénéficient rarement d'une prise en charge par une équipe spécialisée en soins palliatifs (SPs) et meurent généralement à l’hôpital, y compris en unités de soins intensifs (USI). [1, 2].

L'intensité des soins de fin de vie serait liée, d'une part, à l'imprévisibilité de l'évolution pronostique des hémopathies malignes avancées, aux innovations thérapeutiques toujours plus nombreuses, pouvant apporter une "nouvelle chance », mais répondant souvent aux besoins d'espoir des patients aussi bien qu'au sentiment d'échec des hématologues aux prises avec des maladies réfractaires [2, 3].

Le malaise des hématologues face à l'échec thérapeutique et leur crainte de diminuer l'espoir des patients et de leurs proches entraînent une inadaptation de l'information donnée, ainsi qu'un moindre recours à la désignation de la personne de confiance et la rédaction de directives anticipées (DA) [4].

La perception négative des SPs par les hématologues [5], les spécificités des soins hématologiques (notamment l'indisponibilité fréquente de transfusions en unités de soins palliatifs - USP) ${ }^{1}$ et l'insuffisance des structures constituent autant des barrières aux SPs en hématologie [5,7]. Soulignons, néanmoins, que des expériences des consultations avancées en SPs dans le cadre des services d'hématologie décrivent des résultats encourageants [8; 9]

\footnotetext{
1. Voir à ce propos la discussion proposée par To et al [6].
} 
Ceci est d'autant plus relevant que le caractère tardif des discussions sur le pronostic et les décisions d'arrêt du traitement, la situation clinique du patient (possibilité de détérioration soudaine et de décès rapide), tout autant que la disponibilité des ressources (réseaux familiaux, organisation de SPs), peuvent empêcher le décès au domicile qui aurait été souhaité par les patients et les proches [10]. Une prise en charge inadaptée en fin de vie peut avoir retentissements sur l'entourage du patient. Notons à ce propos que le décès en USI est associé à des risques plus élevés de deuil pathologique chez les proches [11].

\section{Allogreffe de cellules souches hématopoïétiques et soins palliatifs}

Dans le cadre de l'allogreffe, malgré les enjeux pronostiques et les risques de morbimotralité inhérents à la procédure, les obstacles à l'information sur le pronostic persistent: seulement la moitié des patients allogreffés abordent effectivement la question du pronostic avec leur médecin [12]. Aussi, les discussions sur les options de traitement ont lieu seulement lorsque l'état de santé du patient se détériore [13].

Attribué aux spécificités de l'allogreffe et à la difficulté d'estimer les facteurs prédictifs d'une évolution péjorative, le caractère tardif de ces discussions entraîne une haute intensité de soins en fin de vie [14], y compris chez les patients en rechute postallogreffe [15].

Les DA sont rarement abordées avec les médecins greffeurs, alors que cette discussion est susceptible de réduire le recours aux soins intensifs (coûteux et souvent inutiles), sans altérer ni l'espoir des patients vis-à-vis de la procédure, ni leur confiance dans l'équipe médicale [16].

Ces discussions sur les soins en fin de vie sont influencées par les perceptions des médecins greffeurs, qui doutent des connaissances des équipes de SPs sur l'allogreffe, aussi bien que de l'acceptation, par les patients, du terme « soins palliatifs » [17].

Autant d'«obstacles culturels » qui portent préjudice à la collaboration entre greffeurs et équipes de SPs, ainsi qu'à la qualité des soins, alors que des effets positifs des SPs sur le contrôle de la symptomatologie physique et psychique (fatigue, qualité de vie, anxiété, dépression, syndrome de stress post-traumatique) chez les patients allogreffés ont été démontrés [18, 19]. Par ailleurs, différentes modalités de coopération entre les SPs et les unités de greffe attestent de leur intérêt [20] 
Il est à noter que les difficultés de prise en charge des patients allogreffés en fin de vie suscitent chez les équipes de greffe une souffrance morale, identifiée comme un des facteurs générateurs de burn out chez ces professionnels [21].

\section{Prise en charge palliative et allogreffe pédiatrique}

La complexité de la prise en charge palliative dans le cadre de l'allogreffe pédiatrique a trait, d'une part, aux attentes vis-à-vis de la procédure et, d'autre part, à la relation du greffeur à l'enfant et aux parents.

Les études menées en oncologie pédiatrique montrent l'importance accordée par les parents à la qualité de l'information sur la pathologie, le traitement et le pronostic de leur enfant. Or, la discussion sur les éléments pronostiques est, dans la plupart des cas, initiée par les parents, probablement du fait de l'embarras ressenti par les pédiatres face aux situations d'annonce de mauvaises nouvelles. C'est pourtant la qualité de la relation avec le pédiatre qui sera le facteur déterminant de la participation des parents aux décisions thérapeutiques et de leur satisfaction vis-à-vis des choix effectués. [22].

Plutôt qu'une décision partagée, le choix de l'allogreffe pédiatrique s'avère être l'acceptation d'une indication médicale [23]. Le même constat est fait en ce qui concerne les prises de décision en fin de vie, l'espoir de guérison restant présent - chez les parents et chez les pédiatres greffeurs - jusqu'au constat de la détérioration de l'état clinique de l'enfant [24].

L'idéalisation de l'allogreffe, seule perspective curative, et l'existence d'alternatives thérapeutiques multiples et innovantes en cas de récidive tendent à nourrir cet espoir de guérison.

En effet, en ce qui concerne la durée de survie, un écart important entre la démarche curative (entre 140 et 392 jours) et la palliative (médiane = 43 jours) a été démontré chez les patients pédiatriques en rechute post-greffe [25]. Cette étude n'aborde pas la question de la qualité de vie des patients au cours de cette période de survie. Soulignons à ce propos qu'une discordance entre les évaluations faites par les parents et l'enfant allogreffé quant à son état psychique et à sa qualité de vie [26].

La poursuite d'une démarche curative amène les patients adolescents et jeunes adultes (AJA) allogreffés à être fréquemment hospitalisés en USI au cours de leur dernier mois de vie [27]. Précisons que les DA rédigées par ces patients consignent leur souhait de bénéficier de tous les soins nécessaires au maintien de la vie. Il revient alors aux 
greffeurs de chercher un équilibre entre le respect des choix des patients et les chances effectives de guérison pour mieux orienter les décisions thérapeutiques [28].

C'est la reconnaissance précoce par les médecins et les parents du caractère infructueux des soins intensifs qui permet l'intervention des SPs pédiatriques, plutôt bien acceptée par les patients et leurs parents, avec un meilleur contrôle des symptômes, le soulagement de la souffrance des enfants et la réduction des décès en USI [29]. Rappelons que les décès en USI sont liés à l'incidence de deuils compliqués chez les parents [30].

\section{Questions posées}

L'adéquation de l'approche palliative dans les soins aux patients allogreffés est questionnée: d'une part, du fait des spécificités de la procédure et des ressources existantes; d'autre part, du fait des représentations des SPs chez les patients et leurs proches, mais aussi chez les médecins greffeurs.

L'association de l'appellation « soins palliatifs » à la fin de vie et l'existence des « soins de support » peuvent constituer un obstacle à l'intégration précoce des SPs dans la prise en charge des patients allogreffés, alors que l'anticipation de situations complexes liées à la morbi-mortalité de la procédure semble pertinente.

Se pose alors le problème de l'information des patients, notamment sur les risques d'échec thérapeutique et sur le caractère péjoratif du pronostic dans certaines situations cliniques (récidive, complications sévères, GVH réfractaire) : à quel moment aborder ce sujet ? Comment encourager le patient à désigner une personne de confiance et à rédiger des DA?

Par ailleurs, émerge la question de la proportionnalité des soins : comment favoriser les discussions pluri-professionnelles à ce sujet ? Quels outils d'aide à la décision? Comment tracer ces discussions afin d'assurer une cohérence dans les actes médicaux, les gestes soignants et les interventions des différents professionnels?

Enfin, les manières de favoriser la collaboration entre les équipes de greffe et les équipes de SPs ont été discutées, la formation initiale et continue des médecins et des paramédicaux étant évoquée comme condition sine qua non.

\section{Méthodologie :}

Cet atelier a été conduit selon la méthodologie des ateliers d'harmonisation des 
pratiques de la SFGM-TC [31], dont l'objectif est de proposer des recommandations qui, adaptées aux réalités des différents centres, visent la standardisation et l'amélioration de la qualité des soins.

Une revue bibliographique a permis de colliger les données concernant la prise en charge palliative des patients allogreffés.

Afin connaître les pratiques courantes dans les unités de greffe, nous avons conçu un questionnaire dirigé aux équipes de greffe (annexe 1); et un deuxième ciblant les professionnels des soins palliatifs (annexe 2).

Le premier questionnaire a été diffusé par mail à trois reprises aux professionnels (médecins et infirmiers) registrés à la SFGM-TC. Ces professionnels ont été invités à transmettre le questionnaire à d'autres soignants impliqués dans les soins aux patients allogreffés. Les membres du groupe de travail se sont également chargés de cette diffusion, ce qui a pu entraîner une surreprésentation de certains centres dans les données recueillies.

Le deuxième questionnaire a été diffusé à deux reprises aux professionnels exerçant en USP, en équipe mobile de soins palliatifs (EMSP) adultes et pédiatriques et réseaux de soins palliatifs pédiatriques à partir de l'annuaire de la Société Française d'accompagnement et soins palliatifs (SFAP), mailing complété par des contacts pris par des membres du groupe de travail.

Soulignons que ces questionnaires n'avaient pas une visée de représentativité ou d'exhaustivité des données, mais d'identifier les facteurs susceptibles de faciliter ou de freiner la prise en charge palliative au sein des centres de greffe.

\section{Résultats :}

\section{Les équipes de greffe}

Cent quatre-vingt-dix professionnels exerçant dans 30 centres de greffe ont répondu au questionnaire, majoritairement infirmiers (44\%), médecins (34,7\%), mais aussi cadres infirmiers, aides-soignants, psychologues, diététiciens, assistants sociaux, kinésithérapeute. Relevons que la majorité des répondants (72\% des infirmiers et aidessoignants et $85 \%$ des médecins) exercent en hématologie depuis plus de 5 ans.

Pour ces professionnels, la question des SPs se pose lorsqu'ils sont confrontés à la rechute post-greffe (79\%), à des maladies réfractaires (79\%), à la maladie du greffon 
versus l'hôte (GVH - 80\%). Néanmoins, 62,7\% des paramédicaux et 80\% des médecins considèrent qu'il n’y a pas des critères établis permettant de décider du passage du curatif au palliatif chez ces patients. La prise en charge palliative suscite, donc, des dilemmes au sein des équipes (79\% des paramédicaux, 86\% des médecins). Du fait de l'incertitude quant à l'évolution de l'état du patient, il est difficile d'aborder la transition du curatif au palliatif (87\%), l'information aux patients et aux familles posant ainsi problème pour les professionnels.

En effet, seul un quart des répondants déclare que la question des DA est abordée avec les patients et leurs familles, alors que la majorité des professionnels considère que ce sujet devrait être systématiquement discuté avant l'allogreffe (lors de la consultation ou du bilan pré-greffe).

Pour $88 \%$ des répondants, les décisions de passage du curatif au palliatif devraient être prises en staff pluridisciplinaire, mais 53,7\% affirment que ces staffs n'ont pas lieu au sein de leurs unités de greffe. Notons que 48\% des médecins ne considèrent pas pertinent de consulter systématiquement les EMSP lors de ces décisions, contrairement à $81 \%$ des paramédicaux, favorables à cette consultation.

De même, nous constatons un écart considérable entre les réponses négatives des paramédicaux $(39,5 \%)$ et positives des médecins $(87,8 \%)$ concernant le travail régulier avec les EMSP. Les données qualitatives éclairent ce constat.

Tous les répondants (excepté un) déclarent avoir besoin de formation en SPs. On relève que les médecins se considèrent suffisamment formés quant aux aspects psychologiques $(70 \%)$ et relationnels $(87,8 \%)$, contre respectivement $34,8 \%$ et $38,7 \%$ des paramédicaux.

Enfin, nous remarquons qu'un tiers des répondants estiment que les patients allogreffés en fin de vie et leur entourage ne bénéficient pas d'un accompagnement psycho-social adapté au sein de leur Service. Là encore, il est à signaler une différence importante entre les perceptions des soignants et celle des médecins, qui sont respectivement 32\% et $16,6 \%$ à se dire insatisfaits de la qualité de cette prise en charge en fin de vie.

\section{Données qualitatives:}

A propos de la prise en charge palliative chez les patients allogreffés, on remarque une différence entre les commentaires des médecins, axés sur la complexité clinique de ces 
situations, et ceux des paramédicaux, plus ciblés sur l'inconfort, l'autonomie, la qualité de vie, «la souffrance psychologique et physique du patient ».

Les paramédicaux mettent en avant un positionnement médical jugé «jusqu'auboutiste » :

«... il y a "trop" souvent un "énième" traitement à proposer pour pallier aux complications nombreuses post-greffe et ce malgré le fait que, d'un point de vue objectif, le patient n'a presque plus de qualité de vie».

Les médecins font valoir «l'évolution imprévisible de la maladie » et l'existence de «certains traitements spécifiques de la maladie, les thérapies ciblées, [qui] permettent de prolonger la durée de vie, parfois avec rémission, parfois sans... le temps permet aussi parfois d'organiser un autre traitement (CAR-T)».

Néanmoins, certains médecins disent la difficulté de "lâcher prise »: "L'allogreffe véhicule l'image d'une procédure salvatrice idéalisée. On y met tout son espoir: pas de place pour un échec... encore plus si c'est la TRM (transplant-related mortality : mortalité liée à la greffe) qui met la vie du patient en danger». On entend là le sentiment de responsabilité, voire de culpabilité, du médecin, l'induisant à « ne pas baisser les bras ».

Reconnaissant ces difficultés, certains défendent l'utilité d'«une discussion sur les DA avant la greffe » et «l'intégration des soins palliatifs dès le début de la maladie ».

Plusieurs répondants regrettent "le manque d'espace "formel" de discussion pluridisciplinaire quand une aggravation chez un patient est constatée ». D'où l'utilité d'un «guide», «pour aborder au mieux ces situations qui mettent tout le monde mal à l'aise ».

En effet, les commentaires font état d'une souffrance au sein des équipes, d'une incompréhension, voire des conflits, entre médecins et équipes soignantes, notamment quant au caractère tardif des discussions avec les patients et l'entourage sur les décisions en fin de vie.

L'intérêt de l'expertise et de l'avis tiers des EMSP lors des discussions sur le passage du curatif au palliatif, évitant ainsi l'écueil de « la charge émotionnelle qui pourrait affecter le jugement décisionnel de la prise en charge», est mentionné. Néanmoins, certains commentaires des médecins mettent en doute la compétence des EMSP en matière d'allogreffe. Probablement de ce fait, le travail régulier avec les EMSP se restreint au seul domaine de la symptomatologie douloureuse. 
Les paramédicaux attribuent l'absence de recours aux EMSP à l'opposition des hématologues qui "aiment gérer "seuls" la prise de décision » et qui «ont l'impression d'abandonner le patient s'ils font appel à l'équipe mobile». Un commentaire évoque un rapport entre la résistance aux SPs et cette appellation: «les médecins comme les patients ont peur de ce mot parce qu'ils pensent que ça veut dire qu'on lâche tout».

Par contre, il apparaît que certains centres ont un «long compagnonnage » avec les EMSP et mettent en avant l'intérêt d' « une collaboration dès le diagnostic ».

Les paramédicaux sont insatisfaits de l'accompagnement psychosocial des patients allogreffés en fin de vie et expriment des regrets quant à des «prises de décision tardives » et au manque de concertation pluri-professionnelle. Les médecins soulignent l'humanité des équipes, mais déplorent le manque de moyens humains (ex : des postes à temps partiel pour les psychologues et assistantes sociales). Ils reconnaissent la difficulté de prendre en charge ces situations de fin de vie, «vécues comme un échec ».

\section{Les équipes des SPs}

Nous avons obtenu 19 réponses exploitables (18 médecins et un cadre de santé), provenant de 15 centres différents. La moitié des participants travaille régulièrement avec les services d'hématologie, et la majorité a été amenée à prendre en charge un patient allogreffé (en moyenne de 3,5 interventions par mois : min 0,5/max 20).

La collaboration entre les équipes de greffe et de SPs

Les interventions concernent la fin de vie des patients en rechute post-allogreffe ou présentant une GVH réfractaire, la gestion de symptômes d'inconfort, la prise en charge de l'entourage et l'aide à la clarification sur le statut palliatif ou non réanimatoire d'un patient greffé.

La concordance entre les attentes des équipes d'allogreffe et les missions des soins palliatifs est jugée bonne par deux tiers des participants; néanmoins, ils auraient aimé intervenir plus précocement dans les prises en charge :

«Il existe un manque d'anticipation du service d'hématologie dans l'introduction de l'équipe de soins palliatifs dans la prise en charge du patient ».

Hospitalisations de patients greffés en USP

Une USP existe au sein du centre hospitalier où exerce la majorité des participants et la moitié d'entre eux a auparavant admis un patient allogreffé dans cette unité. Les 
difficultés signalées ont trait à l'appréhension du patient et de sa famille face à la mise en place de protocoles de soins différents (Paracétamol systématique, seuil transfusionnel). La non-orientation aux USP est attribuée à une réticence de l'équipe de greffe, qui considérerait la prise en charge en USP comme inadaptée à la spécificité des patients allogreffés.

Les participants signalent qu'il n'existe pas de lits d'USP pour les enfants, qui sont hospitalisés dans les lits identifiés dans les services de pédiatrie.

Le rôle des EMSP

Selon les participants, le recours aux EMSP devrait s'envisager pour les raisons suivantes (par ordre de fréquence) : complications amenant à questionner le statut nonréanimatoire ou palliatif du patient (100\% des répondants), présence de symptômes d'inconfort non corrigés par les traitements classiques, organisation d'un retour au domicile complexe pour une prise en charge de fin de vie, statut palliatif posé, GVH réfractaire, rechute post-allogreffe, problèmes avec la famille. L'aide à la réflexion sur la proportionnalité des soins et le soutien aux soignants et aux patients sont aussi mentionnés.

D’après les participants, n'importe quelle personne (patient, famille, équipe paramédicale, équipe médicale ou médecin traitant) peut faire appel à l'EMSP après en avoir discuté et informé le médecin référent.

Discussions collégiales pour limitation et arrêt des traitements actifs (LATA)

La majorité des répondants connait l'organisation de ces réunions, la moitié y participe, un tiers affirme ne pas y être convié. D’après certains répondants, ces discussions ne sont pas systématiques. Les commentaires à ce propos attirent l'attention sur les difficultés de collaboration avec les équipes d'hématologie et le caractère tardif des interventions des EMSP au sein de ces services.

\section{Recommandations}

Nos recommandations sont assorties de quelques documents de travail proposés à titre d'exemple; chaque équipe pouvant juger de leur utilité et les adapter à sa propre organisation.

Démarche palliative précoce 
Dans la mesure où les pathologies hématologiques sont grevées d'un pronostic sombre, et que l'allogreffe est elle-même pourvoyeuse de complications potentiellement létales, les risques d'échec et de complications liées à la procédure doivent toujours être expliqués au patient lors de l'entretien pré-greffe. Les patients doivent être informés du risque de transfert en réanimation, mais aussi de la continuité des soins quelle que soit la situation clinique, incluant la prise en charge palliative. L'intérêt de désigner une personne de confiance, avec qui ils peuvent discuter de leurs souhaits de prise en charge en cas d'impossibilité pour eux-mêmes de s'y prononcer, ainsi que de rédiger des DA doit être évoqué. Il est essentiel d'expliquer aux patients l'utilité de ces documents, en soulignant qu'ils peuvent être revus à tout moment au fil de la prise en charge. Cette information sera appuyée de la remise de documents ad hoc (annexe 3).

L'intervention des soins de support et/ou des EMSP (selon les ressources locales) doit être proposée, et le médecin doit s'efforcer de les démythifier, en s'appuyant sur les données scientifiques sur les bénéfices de ce type d'intervention.

Une prise en charge palliative de qualité nécessite une bonne collaboration entre équipes d’hématologie et de SPs. Les modalités organisationnelles étant variables, il est important de connaître les conditions et possibilités d'intervention des EMSP (voir annexe 4) et les ressources disponibles (possibilités de transfusion, lits dédiés, USP, réseaux de SPs, hospitalisations à domicile - HAD).

Notons que la collaboration avec les EMSP implique le principe de non-substitution, qui assure au médecin référent la responsabilité des prescriptions, lui permettant par conséquent de prendre en compte - ou non, les avis délivrés. Il est fondamental que les personnes impliquées (patient, entourage, équipes de soin) puissent comprendre les modalités de collaboration visant à assurer la cohérence de la prise en charge et la qualité des soins.

Tout au long du parcours d'allogreffe, et particulièrement lors de complications graves, la proportionnalité des soins doit être discutée en équipe pluridisciplinaire, idéalement avec un membre de l'EMSP. Ces discussions donneront lieu à une trace écrite, sur laquelle les différents intervenants pourront s'appuyer, pour assurer la continuité et la cohésion dans les soins (annexes 5, 6, 7).

La gravité de certaines situations est suffisamment connue pour que la question de la proportionnalité des soins et $\mathrm{du}$ niveau d'engagement thérapeutique soit systématiquement soulevée: rechute post-allogreffe, 2ème allogreffe, rejet primaire, 
infections sévères répétées, syndrome d’obstruction sinusoïdal réfractaire, perte d'autonomie, refus des soins invasifs par le patient, GVH réfractaire.

Enfin, les documents supra-cités (désignation de la personne de confiance, DA, fiches de LATA), doivent être renseignés dans le dossier médical du patient.

\section{Pluridisciplinarité}

La prise en charge palliative au sein des unités de greffe implique une approche holistique du soin, de nature médico-psycho-sociale, intégrant la perspective du patient. Cette approche nécessite l'intervention coordonnée de différentes professions soignantes, dont la coopération ne peut se faire sans le partage des finalités du soin. Afin de «faire équipe», nous recommandons la participation interprofessionnelle à des temps de transmission communs, où le sens des soins délivrés sera clairement nommé et partagé, ainsi que la sollicitation des différents professionnels (psychologue, assistant social, kinésithérapeute, ergothérapeute, etc) au lit du patient, aussi bien au sein du service hospitalier qu'au domicile. La démarche pluridisciplinaire doit être anticipée et poursuivie: les différents acteurs au domicile sont coordonnés par les réseaux de soins palliatifs ${ }^{2}$ ou par le médecin traitant, s'ils sont sollicités en amont dans cet objectif par l'unité de greffe.

Nous encourageons également la mise en œuvre d'une culture de la délibération, définie comme «l'activité, pratique et réflexive, qui permet de choisir une orientation jugée préférable dans le soin » [32]. Ceci afin de répondre à la complexité des situations cliniques à travers des temps d'échanges permettant de clarifier les enjeux et les orientations possibles de prise en charge. Le temps de la décision revient ensuite au médecin référent, éclairé par la synthèse de la délibération collective.

Soulignons que la délibération collective n'est pas assimilable à une expertise technique ou scientifique (réunions de concertation pluridisciplinaire) ni réductible au cadre de la procédure collégiale concernant toute décision de LATA.

Enfin, on peut recommander, au sein de la démarche pluridisciplinaire comme de la délibération collective, l'appel aux EMSP qui peuvent jouer un rôle de tiers, de par leur conception différente du soin, de par leur expertise dans le domaine des symptômes complexes et dans l'accompagnement de la personne malade.

\footnotetext{
${ }^{2}$ Carte des réseaux de soins palliatifs au domicile. http://www.sfap.org/annuaire
} 


\section{Formation des équipes}

Les réponses aux questionnaires par les équipes de greffe et de SPs mettent en évidence une méconnaissance mutuelle de ces deux spécialités.

Certains médecins-greffeurs assimilent les SPs à la fin de vie et ne réfèrent pas leurs patients aux USP en considérant qu' «ils ne savent pas s'en occuper ». D'autre part, les équipes d'USP peuvent se trouver en difficulté avec les patients allogreffés, car la gestion des traitements immuno-suppresseurs et des transfusions leur est inhabituelle3.

La formation est donc nécessaire à tous les professionnels impliqués dans la prise en charge des patients allogreffés.

Identifiées comme partenaires, les équipes de SPs pourraient bénéficier des formations en allogreffe prodiguées par les équipes de greffe en réponse au référentiel JACIE.

De même, la formation étant une des missions des équipes de SPs, celles-ci peuvent contribuer à la formation continue des équipes de greffe (médecins et paramédicaux), par exemple sous la forme d'interventions thématiques courtes, au sein des unités. Une intervention est particulièrement souhaitable auprès des internes lors de leur arrivée dans les services de greffe.

Par ailleurs, les SPs doivent faire partie du contenu de la formation médicale initiale, des DES d'Hématologie et des diplômes universitaires, tels que le DU d'Allogreffe. Ces formations doivent comprendre au minimum les aspects législatifs (Loi Clays-Léonetti, DA, sédation terminale), des éléments de communication sur la fin de vie, un volet psycho-social et la formation à la délibération collective.

Les SPs faisant partie des soins courants prodigués aux patients, il est souhaitable de discuter en réunion-qualité de type CREX (Compte-Rendu d'Expérience) ou RMM (Revue de Morbi-Mortalité) une prise en charge palliative jugée insatisfaisante, afin d'améliorer les pratiques professionnelles.

\section{$\underline{\text { Soutien aux équipes }}$}

Les soins en fin de vie sont source de souffrance morale pour les membres des équipes de greffe. Cette souffrance est liée à un écart entre ce qui le professionnel pense être le mieux pour le patient et ce qu'il est en mesure de mettre en acte pour accomplir ce projet de soin. Par ailleurs, des désaccords entre les différents professionnels,

\footnotetext{
3 . Webb et al. [40] résument les connaissances essentielles aux équipes de SPs intervenant en Hématologie.
} 
notamment des écarts entre les positions des équipes médicale et paramédicale, peuvent susciter des sentiments de dévalorisation chez les soignants, des difficultés relationnelles qui pourraient accroître cette souffrance.

La mise en place de staffs pluridisciplinaires reconnus institutionnellement, réguliers, avec l'engagement des responsables du service (cadre de santé, médecin) est la première réponse à cette problématique. Il s'agit d'instaurer une démarche participative, reconnaissant les compétences de chacun, valorisant les différents points de vue, encourageant les échanges, afin d'établir un projet de soin qui redonne sens aux actes soignants de chaque professionnel impliqué dans la prise en charge du patient et dans l'accompagnement de ses proches.

La deuxième réponse, c'est la formation continue : des séances courtes mises en place au sein des unités peuvent favoriser les échanges et contribuer à la construction d'une démarche commune au sein des équipes.

Enfin, d'autres espaces d'échanges, par exemple des groupes Balint [34], peuvent permettre élaboration de la charge émotionnelle suscitée par la prise en charge en fin de vie des patients allogreffés, souvent suivis depuis longtemps par les équipes.

\section{Spécificités des SPs en allogreffe pédiatrique}

Dans le contexte de l'allogreffe pédiatrique, porteuse d'espoir pour les enfants et leurs parents, les pédiatres ont des difficultés à aborder les risques de morbi-mortalité, d'échec thérapeutique et le caractère péjoratif du pronostic.

Ainsi, une première recommandation est de concevoir l'information comme un processus collectif. La notion de processus sous-entend la nécessité de plusieurs rencontres entre le pédiatre référent, les parents et l'enfant ou l'adolescent malade, la charge émotionnelle des informations sur la procédure nécessitant un temps indispensable à leur assimilation. L'idée d'un processus collectif implique l'intégration d'autres professionnels (infirmiers, psychologue, assistant social...), y compris les EMSP pédiatriques, présentés comme membres prenant part aux soins du patient et à l'accompagnement des parents et des proches tout au long de la procédure ${ }^{4}$.

\footnotetext{
4 . Soulignons qu'en nous référant aux "parents », nous n'oublions pas de considérer les différents besoins de la mère et du père en termes d'information et de soutien. Par ailleurs, en nommant les proches, nous souhaitons insister sur les répercussions de l'allogreffe sur la fratrie de l'enfant malade, en particulier sur le donneur.
} 
Dès lors, ces professionnels pourront participer à la réflexion sur la proportionnalité des soins en cas de complications graves, de GVH réfractaire ou de récidive de la maladie. Les décisions prises suite à ces discussions pluri-professionnelles seront consignées dans le dossier du patient afin que tous les professionnels impliqués dans la prise en charge du patient et dans l'accompagnement des parents puissent s'y référer.

Avec l'accord des parents, il semble indispensable de donner la parole à l'enfant, en adaptant le discours médical à son âge et en cherchant à saisir sa perception de la situation. Rappelons qu'un écart a pu être noté entre la perception des parents et celle de l'enfant quant à son état physique et psychique et sa qualité de vie.

La prise en compte de la souffrance de l'enfant ou de l'adolescent peut représenter une limite aux attentes déraisonnables du patient et/ou de ses parents et des équipes médicales.

C'est ainsi que la proposition des SPs prendra sens, avec l'objectif d'un meilleur contrôle de la symptomatologie douloureuse et/ou anxieuse du patient, l'évitement des soins invasifs et la possibilité d'un choix du lieu de prise en charge de la fin de vie (hôpital ou domicile).

Dans le cas de fin de vie au domicile, la collaboration avec les HAD et les réseaux de SPs pédiatriques permettra aux équipes de greffe de poursuivre les soins au patient et l'accompagnement des parents et des proches, en évitant le sentiment d'abandon qui peut être éprouvé dans ces situations.

\section{Questions résiduelles}

Des obstacles organisationnels et culturels à la prise en charge palliative subsistent.

Parmi les obstacles «organisationnels», la problématique des transfusions persiste. Nécessaires au confort des patients en fin de vie, les transfusions réitératives peuvent être un frein au transfert en USP ou à la prise en charge au domicile, notamment du fait des trajets itératifs à l'hôpital, assez peu compatibles avec la qualité de vie. Précisons que la possibilité d'accéder aux transfusions varie selon les USP et que plusieurs HAD essaient de faire évoluer cette situation et de proposer à l'avenir des transfusions à domicile. Ces avancées sur le plan organisationnel n'évincent pas la nécessité d'une réflexion sur la limitation transfusionnelle en toute fin de vie. 
Le problème du forfait journalier de prise en charge des patients peut être un autre obstacle au retour à domicile. Effectivement, le patient nécessitant des traitements onéreux peut ne pas être éligible à une HAD ou à un Service de soins de suite et de rééducation (SSR) du fait du coût de la prise en charge. L'inscription des médicaments sur la liste en sus peut minimiser cette problématique qui doit, toutefois, être prise en compte au niveau des hautes instances sanitaires.

Au-delà de ces aspects organisationnels, des « obstacles culturels » sont bien présents dans les pratiques actuelles, marquées par l'insuffisance, voire l'absence, des staffs pluriprofessionnels et le caractère tardif des discussions sur les soins en fin de vie.

Alors que nous conseillons d'aborder précocement les notions d'échec possible et de prise en charge palliative, il est difficile de désigner un moment précis pour ce dialogue, du fait même de la diversité de l’organisation du parcours pré-greffe. Souvent réalisés le jour du bilan, où de nombreux examens sont effectués, les entretiens médicaux de greffe, déjà très denses en information, sont parfois suivis de l'entretien infirmier.

Mais au-delà de la densité des informations présentées et des contraintes organisationnelles, l'obstacle principal reste la réticence des médecins à parler d'échec, de fin de vie, alors même qu'ils présentent une thérapeutique qui se veut curative. Notons que ces questions peuvent être évoquées lorsque le médecin-greffeur mentionne les risques de passage en réanimation, en introduisant alors des documents sur la désignation de la personne de confiance et les DA.

Une évolution de ces pratiques nécessite donc un changement des mentalités et une amélioration des capacités communicationnelles, qui doivent être favorisés par la formation continue de tous les professionnels impliqués dans la prise en charge des patients allogreffés.

Soulignons que dans certains centres la collaboration avec les EMSP se fait de façon régulière et des initiatives, telles que les consultations avancées en SPs, majoritairement bien perçues par les patients, pourraient être démultipliées.

\section{Déclaration de liens d'intérêts :}

Les auteurs ne déclarent pas de conflit d'intérêt

La SFGM-TC remercie les partenaires industriels pour leur soutien financier qui ont permis la réussite de cette onzième édition des ateliers d'harmonisation des pratiques : ACCORD, AMGEN, ASTELLAS, BIOTEST, BLUEBIRDBIO, INCYTE, JAZZ PHARMACEUTICALS, MACOPHARMA, MALLINCKRODT THERAKOS, MSD FRANCE, SANOFI GENZYME. 


\section{Références bibliographiques}

[1] El-Jawahri A, Nelson AM, Gray TF, Lee SJ, LeBlanc TW. Palliative and End-of-Life Care for Patients With Hematologic Malignancies. J Clin Oncol. 2020 20;38(9):944-953.

[2] Beaussant Y, Daguindau E, Chauchet A, Rochigneux P, Tournigand C, Aubry R, Lucas Morin L. Hospital end-of-life care in haematological malignancies. BMJ Support Palliat Care 2018; 8(3):314-324.

[3] Odejide O, Cronin AM, Condron N, Earle CC, Wolfe J , Abel GA. Timeliness of Endof-Life Discussions for Blood Cancers: A National Survey of Hematologic Oncologists. JAMA Intern Med. 2016; 176(2):263-5.

[4] Gray TF, Temel JS, El-Jawahri A. Blood Rev. 2020 Apr 6;100692. online ahead off print. doi: 10.1016/j.blre.2020.100692

[5] Hui D, Park M, Liu D, Reddy A, Dalal S, Bruera E. Attitudes and Beliefs Toward Supportive and Palliative Care Referral Among Hematologic and Solid Tumor Oncology Specialists. Oncologist. 2015; 20(11):1326-32.

[6] To THM, LeBlanc TW, Eastman P, Neoh K, Agar MR, To LB et al. The Prospective Evaluation of the Net Effect of Red Blood Cell Transfusions in Routine Provision of Palliative Care. J Palliat Med. 2017; 20(10):1152-1157.

[7] Odejide O, Cronin AM, Earle CC, Tulsky JA, Abel GA. Why are patients with blood cancers more likely to die without hospice? Cancer 2017; 123 (17): 3377-3384.

[8] Prod'homme C. Mise en place d'une consultation médicale en soins palliatifs dans un service d'hématologie. Retour et réflexion critique sur un an de pratique. Bull Cancer 2020; 107 (11): 1118-1128

[9] Selvaggi KJ, Vick JB, Jessell SA, Lister J, Abraham JL, Bernacki R. et al: Bridging the gap: A palliative care consultation service in a hematological malignancy-bone marrow transplant unit. J Community Support Oncol $2014 ; 12: 50-55$.

[10] McCaughan D, Roman E, Smith AG, Garry AC, Johnson MJ, Patmore RD et al. Perspectives of bereaved relatives of patients with haematological malignancies concerning preferred place of care and death: A qualitative study. Palliat Med. 2019; 33(5):518-530. 
[11] Wright AA, Keating NL, Balboni TA, Plac Matulonis UA, Block SD, Prigerson HG. Place of death: correlations with quality of life of patients with cancer and predictors of bereaved caregivers' mental health. J Clin Oncol 2010; 28: 4457-64.

[12] El-Jawahri A, Traeger L, Kuzmuk K, Eusebio J, Vandusen H, Keenan T et al. Prognostic understanding, quality of life and mood in patients undergoing hematopoietic stem cell transplantation. Bone Marrow Transplant2015; 50: 1119-24.

[13] Tee ME, Balmaceda GZ, Granada MA, Fowler CS, Payne JK. End-of-Life Decision Making in Hematopoietic Cell Transplantation Recipients. December Clin J of Oncol Nurs. 2013;17 (6) 640-646.

[14] Johnston EE, Muffly L, Alvarez E, Saynina O, Sanders LM, Bhatia S, Chamberlain LJ. End-of-Life Care Intensity in Patients Undergoing Allogeneic Hematopoietic Cell Transplantation: A Population-Level Analysis. J Clin Oncol 2018; 36:3023-3030.

[15] Langston JA, Sundaram V, Periyakoil VS, Muffly L. Healthcare Utilization is High in Adult Patients Relapsing after Allogeneic Hematopoietic Cell Transplantation. Biol Blood Marrow Transplant 2019; 25: 1659-1665.

[16] Cappell K, Sundaram V, Park A, Shiraz P, Gupta R, Jenkins P et al. Advance directive utilization is associated with less aggressive end-of-life care in patients undergoing allogeneic hematopoietic cell transplantation. Bone Marrow Transplant. 2018; 24:10351040.

[17] El-Jawahri A, LeBlanc T, Burns LJ, Denzen E, Meyer C, Mau LW et al. What Do Transplant Physicians Think about Palliative Care? A National Survey Study. Cancer. 2018; 124(23): 4556-4566.

[18] El-Jawahri A, LeBlanc T, VanDusen H, Traeger L, Greer JA, Pirl WF et al. Effect of Inpatient Palliative Care on Quality of Life 2Weeks After Hematopoietic Stem Cell Transplantation: A Randomized Clinical Trial. JAMA. 2016; 316(20): 2094-2103.

[19] El-Jawahri A, Traeger L, Greer JA, VanDusen H, Fishman SR, LeBlanc TW et al. Effect of inpatient palliative care during hematopoietic stem-cell transplant on psychological distress 6 months after transplant: Results of a randomized clinical trial. J Clin Oncol 2017 35:3714-3721.

[20] Levine DR, Baker JN, Wolfe J, Lehmann LE, Ullrich C. Strange bedfellows no more: how integrated stem-cell transplantation and palliative care programs can together improve end-of-life care. J Oncol Pract. 2017;13: 569-577. 
[21] Neumann JL, Mau L-W, Virani S, Denzen EM, Boyle DA, Boyle NJ et al. Burnout, Moral Distress, Work-Life Balance, and Career Satisfaction among Hematopoietic Cell Transplantation Professionals. Biol Blood Marrow Transplant 2018 ; 24 :849-860.

[22] Sisk B, Mack J, Ashworth R, DuBois J. Communication in pediatric oncology: State of the field and research agenda. Pediatr Blood Cancer. 2018; 65(1):1-10.

[23] Pentz RD, Pelletier W, Alderfer MA, Stegenga K, Fairclough DL, Hinds PS. Shared Decision-Making in Pediatric Allogeneic Blood and Marrow Transplantation: What If There Is No Decision to Make? The Oncologist 2012;17: 881-885.

[24] Ullrich CK, Dussel V, Hilden JM, Sheaffer JW, Lehmann L, Wolfe J. End-of-life experience of children undergoing stem cell transplantation for malignancy: parent and provider perspectives and patterns of care. Blood. 2010; 115(19): 3879-85.

[25] Roux C, Tifratene K, Socié G, Galambrun C, Bertrand Y, Rialland F et al. Outcome after failure of allogeneic hematopoietic stem cell transplantation in children with acute leukemia: a study by the Société Francophone de Greffe de Moelle et de Thérapie Cellulaire (SFGM-TC) Bone Marrow Transplantation 2017 ; 52, 678-682.

[26] Chang G, Ratichek SJ, Recklitis C, Syrjala K, Patel SK, Harris L. et al. Children's psychological distress during pediatric HSCT: parent and child perspectives. Pediatric Blood \& Cancer 2012; 58(2):289-296.

[27] Snaman JM, Talleur AC, Lu J, Levine DR, Kaye EC, Sykes A et al. Treatment Intensity and Symptom Burden in Hospitalized Adolescent and Young Adult Hematopoietic Cell Transplant Recipients at the End of Life Bone Marrow Transplant. 2018; 53(1):84-90.

[28] Needle J, Smith AR. The Impact of Advance Directives on End-of-Life Care for Adolescents and Young Adults Undergoing Hematopoietic Stem Cell Transplant J Palliat Med 2016 ; 19(3): 300-305.

[29] Ullrich CK, Lehmann L, London WB, Guo D, Sridharan M, Koch R, et al. End-of-Life Care Patterns Associated with Pediatric Palliative Care among Children Who Underwent Hematopoietic Stem Cell Transplant. Biology of Blood and Marrow Transplantation 2016; 22(6): 1049-55.

[30] Meert KL, Shear K, Newth CJ, Harrison R, Berger J, Zimmerman J, et al. Follow-up study of complicated grief among parents eighteen months after a child's death in the pediatric intensive care unit. Journal of palliative medicine. 2011; 14(2):207-14.

[31] Tipton R, Ibrahim Yakoub-Agha I. Ateliers d'harmonisation des pratiques de la SFGM-TC Bull Cancer 2016; 103: S193-S197. 
[32] Mallet D, Begat N, Duchêne V. Déliberer. In: Le Berre R. Eds. Manuel de soins palliatifs. 5 ème édition. Dunod. 2020: 145-161.

[33] Webb JA, Foxwell AM, Jones CA, El-Jawahri A, Kamal AH, Kayastha N et al. Top Ten Tips Palliative Care Clinicians Should Know About Caring for Patients with Hematologic Malignancies. J Palliat Med. 2019;22(11):1449-1454.

[34] Bar-Sela G Lulav-Grinwald D Mitnik I "Balint group" meetings for oncology residents as a tool to improve therapeutic communication skills and reduce burnout level.sêf Cancer Educ. 2012; 27: 786-789 